\title{
Dietary fibre and mortality risk in patients on peritoneal dialysis
}

\author{
Xiao Xu ${ }^{1,2,3}$, Ziqian $\mathrm{Li}^{1,2,3}$, Yuan Chen ${ }^{1,2,3}$, Xihui Liu ${ }^{4}$ and Jie Dong ${ }^{1,2,3 *}$ \\ ${ }^{1}$ Renal Division, Department of Medicine, Peking University First Hospital, Beijing, People's Republic of China \\ ${ }^{2}$ Institute of Nephrology, Peking University, Beijing, People's Republic of China \\ ${ }^{3}$ Key Laboratory of Renal Disease, Ministry of Health and Key Laboratory of Renal Disease, Ministry of Education, Beijing, \\ People's Republic of China \\ ${ }^{4}$ Linyi People's Hospital, Shandong, People's Republic of China
}

(Submitted 14 March 2019 - Final revision received 19 June 2019 - Accepted 12 July 2019)

\section{Abstract}

Higher fibre intake reduced all-cause and cardiovascular mortality among healthy population, but such data in dialysis patients are limited. We aimed to examine these associations in patients on peritoneal dialysis (PD). This single-centre prospective cohort study enrolled 881 incident PD patients between October 2002 and August 2014. All patients were followed until death, transfer to haemodialysis, renal transplantation or until being censored in June 2018. Demographic data were collected at baseline. Biochemical, dietary and nutrition data were examined at baseline and thereafter at regular intervals to calculate the average values throughout the study. The outcomes were defined as all-cause and cardiovascular death. Cox proportional regression models were applied to explore the relationship between fibre intake and outcomes. Participants with higher fibre intake were more likely to be younger, male and have better residual renal function and serum lipids at baseline. They were prone to maintain better nutrient status, higher blood pressure and lower inflammatory status at baseline and afterwards. Neither baseline nor time-averaged fibre intake did show protective effects on all-cause mortality after multivariate adjustment in the whole cohort. Among non-diabetic PD patients, an independent association between fibre intake and all-cause mortality was found, in which each $1 \mathrm{~g} / \mathrm{d}$ increase in time-averaged fibre intake correlated to $13 \%$ of reduction in all-cause mortality. We did not observe any benefits of fibre intake in the CVD mortality for both whole cohort and subgroups. The present study revealed that higher dietary fibre intake appeared to have a protective effect on all-cause mortality in non-diabetic PD patients, which suggest that PD patients should be encouraged to eat a diet rich in fibres.

Keywords: Dietary fibre: Dietary nutrients: Chronic kidney disease: Peritoneal dialysis: Mortality

In the general population, considerable epidemiological evidences have proven that dietary fibre is related to the decreased risk of various chronic diseases such as CVD, type 2 diabetes, hypertension, major cancer ${ }^{(1)}$ and chronic kidney disease (CKD) ${ }^{(2-6)}$. A higher fibre intake is also independently associated with all-cause and CVD mortality in the general population, and among patients with CVD, diabetes mellitus (DM), and cancer, as shown from prospective cohort studies and meta-analysis ${ }^{(7-9)}$.

For patients with CKD, the predictive role of dietary fibre in mortality has been reported from the Uppsala Longitudinal Study of Adult Men (ULSAM) ${ }^{(10)}$ and National Health and Nutrition Examination Survey III (NHANES III) cohorts ${ }^{(11)}$. There are still limited data on the relationship between dietary fibre and mortality in dialysis population, in spite of the recent observational studies indicating that fibre supplementation could improve the lipid profile and oxidative status and decrease the systemic inflammatory state in patients on dialy$\operatorname{sis}^{(12,13)}$. The current guidelines also make little or no reference to dietary fibre intake for patients with CKD due to the lack of evidence ${ }^{(14-16)}$. Recently, a meta-analysis in CKD assessed the benefits of whole dietary pattern in the decreased risk of mortality rather than the nutrient components of the $\operatorname{diet}^{(17)}$. The so-called healthy dietary pattern, enriched with fruit and vegetables, fish, legumes, cereals, whole grains and fibre, cannot recommend the target for a specific nutrient to meet patients' needs.

Therefore, in the present study, we aimed to evaluate the association between dietary fibre intake measured at baseline or in a time-averaged manner and the all-cause and CVD mortality through a prospective peritoneal dialysis (PD) cohort.

Abbreviations: Alb, albumin; CKD, chronic kidney disease; DBP, diastolic blood pressure; DM, diabetes mellitus; HD, haemodialysis; HR, hazard ratio; hs-CRP, high-sensitive C-reactive protein; iPTH, intact parathyroid hormone; PD, peritoneal dialysis; RRF, residual renal function.

* Corresponding author: J. Dong, fax +86010 66551055, email jie.dong@bjmu.edu.cn 
The prognostic value of dietary fibre would also be explored among patients categorised as CVD or non-CVD, DM or non-DM.

\section{Subjects and methods}

Subjects and follow-up

This is a prospective cohort with data retrospectively analysed, and the present study was carried out at the PD centre of Peking University First Hospital. Between 1 October 2002 and 31 August 2014, all incident PD patients were screened. Patients were excluded if they refused to complete the baseline test, denied the diagnosis of end-stage renal disease or could not be regularly followed. All patients were followed until death, transfer to haemodialysis (HD), renal transplantation, loss to follow-up or 30 June 2018 (the end of study). All patients were treated with continuous ambulatory PD and visited a physician at least once every 3 months. All patients began the PD programme within 1 month after catheter implantation and were given lactatebuffered glucose dialysate with a twin-bag connection system (Baxter Healthcare). The present study was approved by the Medical Ethics Committee of Peking University. Written informed consent was obtained from each patient.

\section{Data collection}

Demographic and clinical data including age, sex, BMI, the presence of CVD and DM were collected within the week preceding PD catheter implantation. Baseline values included all measurements of blood pressure, biochemistry, dialysis adequacy, dietary and nutrition parameters in the first 3 months. Baseline values of dietary nutrients were calculated in the first 6 months. All the above measurements during the study period were prospectively collected and averaged for each 6-month interval to calculate the time-averaged values.

\section{Dietary variables}

During the follow-up, patients completed 3-d dietary records before they visited the dietician. A dedicated dietician checked the diary using food models. The dietary records would be invalid if they were recorded in less than $3 \mathrm{~d}$ or did not get checked successfully by the dietitian. Food models were used to estimate the actual amount of foods recorded in the diet diary. Daily fibre, protein, energy, carbohydrate and fat were calculated using a computer software program (PD information Management System, Peritoneal Dialysis Center, Peking University). Oral nutrition supplements including nutritionally complete food product such as Ensure and nutritionally incomplete food product such as protein powder were also recorded to calculate the total amount of protein and energy intake. The daily energy intake includes intake from dietary and dialysate sources.

\section{Biochemical, dialysis adequacy and nutrition variables}

Biochemistry data including $\mathrm{Hb}$, serum albumin (Alb), lipid spectrum, glucose, uric, urea, creatinine, $\mathrm{Ca}$, phosphate, intact parathyroid hormone (iPTH) and so on were examined using an automatic Hitachi chemistry analyzer (Hitachi Chemical). Serum high-sensitive C-reactive protein (hs-CRP) was measured by immune rate nephelometric analysis. Dialysis adequacy, residual renal function (RRF) and glucose absorption were measured by collecting 24-h urine and dialysate. Dialysis adequacy was defined as total urea clearance and total creatinine clearance. Residual renal function was estimated using the average renal clearance of urea and creatinine. Glucose absorption via dialysate was calculated by subtracting glucose amounts in drained dialysate from that in instilled dialysate, expressed as g glucose/d and then the dialysate energy absorption was calculated as $\mathrm{kJ}$ of energy/d.

\section{Definition of outcome event}

The outcomes were defined as cardiovascular and all-cause death. Cardiovascular death is caused due to myocardial infarction, congestive heart failure, cerebral bleeding, cerebral infarction, arrhythmia and peripheral arterial disease ${ }^{(18)}$.

In all analyses, we censored follow-up at transferring to HD, renal transplantation, loss to follow-up or the end of the study (30 June 2018).

\section{Statistical analysis}

The mortality rate in PD cohorts over an average follow-up of 45 months was estimated as $40 \%$ based on our previous research $^{(19,20)}$. The standard deviation of fibre intake in HD patients was $5 \mathrm{~g} / \mathrm{d}^{(21,22)}$ and the expected hazard radio of fibre intake in PD patients was estimated to be $0.95^{(11)}$, according to previous research. We estimated that with a sample of 421 participants, the study would have $90 \%$ statistical power at a significance level of 0.05 for a two-sided test. In order to achieve the greatest statistical power, we included all individuals in the cohort. Statistical analyses were performed using the SPSS software package version 24.0 (SPSS). Parametric data are presented as means and standard deviations. Non-parametric data are presented as median values and interquartile ranges. Categorical variables are expressed as percentages or ratios. Baseline fibre intake and time-averaged fibre intake were categorised by tertile based on the distribution among the study population. One-way ANOVA, Kruskal-Wallis or the $\chi^{2}$ test was used to compare the differences in variables between groups. Differences in the survival curves between the three groups were evaluated using the log rank test. Changes in blood pressure, renal function and laboratory data over time were also compared among groups using a mixed model analysis of variance, with bootstrap covariance accounting for correlated measures within a subject adjusted for baseline characteristics, age, sex, and BMI. Recognised confounders combined with the baseline and timeaveraged fibre intakes were evaluated by the Cox proportional regression model to determine the risk of CVD and all-cause mortality. When the baseline fibre intake was examined, the covariates included age, sex, BMI, baseline mean arterial pressure, Alb, Hb, hs-CRP, TAG, iPTH, RRF, total protein intake and total energy intake; when the time-averaged fibre intake was examined, the covariates included age, sex, time-averaged BMI, mean arterial pressure, Alb, Hb, hs-CRP, TAG, iPTH, RRF, total protein intake and total energy intake. We reported the multivariable-adjusted hazard ratios (HR) with $95 \%$ CI. The calculation of time-averaged biochemistry, nutrition and dialysis parameters in the models was made using the half-yearly 
Nes British Journal of Nutrition

Table 1. Baseline clinical characteristics of peritoneal dialysis (PD) patients ( $n 881)$

(Mean values and standard deviations; numbers of patients and percentages; medians with upper and lower quartiles)

\begin{tabular}{|c|c|c|c|c|c|c|c|c|c|}
\hline \multirow[b]{3}{*}{ Characteristic } & & & \multicolumn{6}{|c|}{ Tertile of fibre intake } & \multirow[b]{3}{*}{$P$} \\
\hline & \multicolumn{2}{|c|}{ Total } & \multicolumn{2}{|c|}{ Low $(<6.4 \mathrm{~g} / \mathrm{d})$} & \multicolumn{2}{|c|}{ Middle $(6.4-9.1 \mathrm{~g} / \mathrm{d})$} & \multicolumn{2}{|c|}{$\operatorname{High}(>9.1 \mathrm{~g} / \mathrm{d})$} & \\
\hline & Mean & SD & Mean & SD & Mean & SD & Mean & SD & \\
\hline Age (years) & 57.7 & 14.8 & $61 \cdot 2^{\star}$ & $15 \cdot 5$ & $57.3 \dagger$ & $14 \cdot 8$ & $54.7 \ddagger$ & $13 \cdot 3$ & $<0.001$ \\
\hline Male & & & & & & & & & \\
\hline$n$ & \multicolumn{2}{|c|}{434} & \multicolumn{2}{|c|}{$111^{*}$} & \multicolumn{2}{|c|}{$142 \dagger$} & \multirow{2}{*}{\multicolumn{2}{|c|}{$\begin{array}{l}181 \\
61.6\end{array}$}} & $<0.001$ \\
\hline$\%$ & & & & & & & & & \\
\hline $\mathrm{BMI}\left(\mathrm{kg} / \mathrm{m}^{2}\right)$ & $23 \cdot 3$ & $3 \cdot 7$ & $22 \cdot 9^{\star}$ & 3.8 & 23.4 & 3.9 & $23 \cdot 6 \ddagger$ & $3 \cdot 3$ & 0.033 \\
\hline \multicolumn{10}{|l|}{ DM } \\
\hline$n$ & \multicolumn{2}{|c|}{371} & \multicolumn{2}{|c|}{133} & \multicolumn{2}{|c|}{125} & \multicolumn{2}{|c|}{113} & 0.230 \\
\hline$\%$ & \multicolumn{2}{|c|}{$42 \cdot 1$} & \multicolumn{2}{|c|}{45.4} & \multicolumn{2}{|c|}{42.5} & \multicolumn{2}{|c|}{38.4} & \\
\hline CVD & & & & & & & & & \\
\hline $\begin{array}{l}n \\
\%\end{array}$ & & & & & & & & & $<0.001$ \\
\hline $\mathrm{SBP}(\mathrm{mmHg})$ & 135.8 & $16 \cdot 7$ & $136 \cdot 0$ & $18 \cdot 4$ & 135.0 & $15 \cdot 4$ & $136 \cdot 4$ & $16 \cdot 2$ & 0.573 \\
\hline $\mathrm{DBP}(\mathrm{mmHg})$ & 79.0 & $11 \cdot 2$ & $77 \cdot 1$ & 11.4 & $78.6 \dagger$ & $10 \cdot 7$ & $81.5 \ddagger$ & $11 \cdot 1$ & $<0.001$ \\
\hline MAP $(\mathrm{mmHg})$ & 98.0 & $11 \cdot 2$ & $96 \cdot 8$ & 11.7 & $97.4 \dagger$ & $10 \cdot 6$ & $100 \cdot 1 \ddagger$ & $10 \cdot 8$ & 0.002 \\
\hline Laboratory and nutrition data & & & & & & & & & \\
\hline Albumin (g/l) & 35.4 & 4.6 & $34 \cdot 6^{*}$ & 4.5 & $35.3 \dagger$ & 4.6 & $36 \cdot 2 \ddagger$ & 4.6 & $<0.001$ \\
\hline $\mathrm{Hb}(\mathrm{g} / \mathrm{l})$ & $102 \cdot 8$ & $15 \cdot 6$ & $100 \cdot 6$ & $15 \cdot 4$ & $101 \cdot 7 \dagger$ & 15.8 & $106.0 \ddagger$ & $15 \cdot 3$ & $<0.001$ \\
\hline hs-CRP (mg/l) & & & & & & & & & \\
\hline Median & & & & & & & & & $<0.001$ \\
\hline Upper and lower quartiles & & & & & & & & & \\
\hline Urea N (mmol/l) & 22.5 & $6 \cdot 1$ & $21 \cdot 1^{*}$ & $6 \cdot 0$ & $22.4 \dagger$ & 5.8 & $24.0 \ddagger$ & $6 \cdot 3$ & $<0.001$ \\
\hline Serum creatinine $(\mu \mathrm{mol} / \mathrm{l})$ & $689 \cdot 2$ & 233.6 & $665 \cdot 0$ & 238.1 & 691.6 & $229 \cdot 4$ & 710.7 & 231.9 & 0.060 \\
\hline Serum $\mathrm{Ca}(\mathrm{mmol} / \mathrm{l})$ & $2 \cdot 2$ & 0.2 & $2 \cdot 2$ & 0.2 & $2 \cdot 2$ & 0.2 & $2 \cdot 2$ & 0.2 & 0.145 \\
\hline Serum $\mathrm{P}(\mathrm{mmol} / \mathrm{l})$ & 1.6 & 0.4 & 1.5 & 0.4 & 1.6 & 0.4 & 1.6 & 0.3 & 0.132 \\
\hline Serum $\mathrm{K}(\mathrm{mmol} / \mathrm{l})$ & 4.4 & 0.6 & $4.3^{*}$ & 0.6 & $4.4 \dagger$ & 0.6 & $4.6 \ddagger$ & 0.6 & $<0.001$ \\
\hline Serum $\mathrm{Na}(\mathrm{mmol} / \mathrm{l})$ & $139 \cdot 2$ & 3.0 & $138 \cdot 8^{*}$ & $2 \cdot 7$ & 139.4 & 3.7 & $139 \cdot 4 \ddagger$ & 2.5 & 0.014 \\
\hline HDL-cholesterol (mmol/l) & $1 \cdot 1$ & 0.3 & $1 \cdot 1$ & 0.3 & $1 \cdot 2$ & 0.4 & 1.1 & 0.3 & 0.874 \\
\hline LDL-cholesterol (mmol/l) & $2 \cdot 6$ & 0.8 & $2 \cdot 6$ & 0.8 & 2.6 & 0.8 & $2 \cdot 6$ & 0.9 & 0.830 \\
\hline Total cholesterol $(\mathrm{mmol} / \mathrm{l})$ & 4.9 & $1 \cdot 1$ & $5 \cdot 0$ & 1.1 & $4.9 \dagger$ & $1 \cdot 2$ & $4.7 \ddagger$ & $1 \cdot 2$ & 0.036 \\
\hline TAG $(\mathrm{mmol} / \mathrm{l})$ & & & & & & & & & \\
\hline Median & & & & & & & & & 0.007 \\
\hline Upper and lower quartiles & & & & & & & & & \\
\hline iPTH (pg/ml) & & & & & & & & & \\
\hline Median & & & & & & & & & 0.007 \\
\hline Upper and lower quartiles & & & & & & & & & \\
\hline Total CCr (litres/week per $1.73 \mathrm{~m}^{2}$ ) & $72 \cdot 8$ & 27.9 & 70.5 & 25.5 & 71.8 & $28 \cdot 0$ & $76.0 \ddagger$ & 29.8 & 0.049 \\
\hline Total Kt/V & 1.9 & 0.5 & 1.9 & 0.5 & 1.9 & 0.5 & 1.9 & 0.6 & 0.688 \\
\hline $\mathrm{RRF}(\mathrm{ml} / \mathrm{min})$ & & & & & & & & & \\
\hline Median & & & & & & & & & 0.001 \\
\hline Upper and lower quartiles & & & & & & & & & \\
\hline Total energy intake $(\mathrm{kJ} / \mathrm{d})$ & $6949 \cdot 2$ & 1398.7 & $6037 \cdot 5^{\star}$ & $1097 \cdot 0$ & $6936.2 \dagger$ & 1114.2 & $7867.6 \ddagger$ & 1325.5 & $<0.001$ \\
\hline Total protein intake $(\mathrm{g} / \mathrm{d})$ & $52 \cdot 1$ & $13 \cdot 9$ & $44 \cdot 1^{*}$ & $11 \cdot 0$ & $51 \cdot 3 \dagger$ & $11 \cdot 0$ & $61.0 \ddagger$ & 13.9 & $<0.001$ \\
\hline Total carbohydrate intake $(\mathrm{g} / \mathrm{d})$ & $186 \cdot 7$ & $52 \cdot 4$ & $146 \cdot 3^{\star}$ & 34.9 & $186 \cdot 3 \dagger$ & 35.6 & $227 \cdot 4 \ddagger$ & 49.8 & $<0.001$ \\
\hline Total fat intake $(\mathrm{g} / \mathrm{d})$ & 54.0 & 14.5 & $47 \cdot 3^{*}$ & $12 \cdot 6$ & $55 \cdot 1 \dagger$ & 14.5 & $59.7 \ddagger$ & 13.7 & $<0.001$ \\
\hline Total fibre intake $(\mathrm{g} / \mathrm{d})$ & 8.2 & 3.4 & $4.9^{*}$ & $1 \cdot 2$ & $7.8 \dagger$ & 0.8 & $11.8 \ddagger$ & 2.8 & $<0.001$ \\
\hline $\mathrm{nDPI}(\mathrm{g} / \mathrm{kg}$ per $\mathrm{d})$ & 0.85 & 0.2 & $0.76^{\star}$ & 0.2 & $0.83 \dagger$ & 0.2 & $0.94 \ddagger$ & 0.2 & $<0.001$ \\
\hline $\mathrm{nDEI}(\mathrm{kJ} / \mathrm{kg}$ per d) & $119 \cdot 2$ & $22 \cdot 6$ & $107 \cdot 9^{\star}$ & 20.9 & $119.7 \dagger$ & 20.5 & $130 \cdot 1 \ddagger$ & 20.9 & $<0.001$ \\
\hline
\end{tabular}

DM, diabetes mellitus; SBP, systolic blood pressure; DBP, diastolic blood pressure; MAP, mean arterial pressure; hs-CRP, high-sensitive C-reactive protein; iPTH, intact parathyroid hormone; Total CCr, total creatinine clearance; Total Kt/ , total urea clearance; RRF, residual renal function; $\mathrm{nDPI}$, normalised protein intake; $\mathrm{nDEl}$, normalised energy intake.

* $P<0.05$ low-tertile group compared with the middle-tertile group.

$+P<0.05$ middle-tertile group compared with the high-tertile group.

$\ddagger P<0.05$ high-tertile group compared with the low-tertile group. 
Table 2. Baseline clinical characteristics of patients who ceased and maintained peritoneal dialysis (PD) ( $n$ 881)

(Mean values and standard deviations; numbers of patients and percentages; medians with upper and lower quartiles)

\begin{tabular}{|c|c|c|c|c|c|c|c|}
\hline \multirow[b]{2}{*}{ Characteristic } & \multicolumn{2}{|c|}{ Total } & \multicolumn{2}{|c|}{ Ceased $(n 730)$} & \multicolumn{2}{|c|}{ Maintained PD ( $n$ 151) } & \multirow[b]{2}{*}{$P$} \\
\hline & Mean & SD & Mean & SD & Mean & SD & \\
\hline Age (years) & $57 \cdot 7$ & $14 \cdot 8$ & $58 \cdot 8$ & 14.9 & $52 \cdot 4$ & $13 \cdot 0$ & $<0.001$ \\
\hline \multicolumn{8}{|l|}{ Male } \\
\hline$n$ & \multirow{2}{*}{\multicolumn{2}{|c|}{$\begin{array}{l}434 \\
49 \cdot 3\end{array}$}} & \multirow{2}{*}{\multicolumn{2}{|c|}{$\begin{array}{l}375 \\
51.2\end{array}$}} & \multicolumn{2}{|c|}{$\begin{array}{c}59 \\
39.1\end{array}$} & 0.006 \\
\hline$\%$ & & & & & & & \\
\hline BMI $\left(\mathrm{kg} / \mathrm{m}^{2}\right)$ & 23.3 & 3.7 & 23.4 & 3.7 & $22 \cdot 9$ & 3.7 & 0.222 \\
\hline \multicolumn{8}{|l|}{ DM } \\
\hline$n$ & \multicolumn{2}{|c|}{371} & \multicolumn{2}{|c|}{330} & \multicolumn{2}{|c|}{43} & \multirow[t]{2}{*}{$<0.001$} \\
\hline$\%$ & \multicolumn{2}{|c|}{$42 \cdot 1$} & \multicolumn{2}{|c|}{$45 \cdot 0$} & & & \\
\hline \multicolumn{8}{|l|}{ CVD } \\
\hline$n$ & \multirow{2}{*}{\multicolumn{2}{|c|}{$\begin{array}{l}375 \\
42 \cdot 6\end{array}$}} & \multirow{2}{*}{\multicolumn{2}{|c|}{$\begin{array}{l}339 \\
46 \cdot 2\end{array}$}} & \multirow{2}{*}{\multicolumn{2}{|c|}{$\begin{array}{c}36 \\
23.8\end{array}$}} & $<0.001$ \\
\hline$\%$ & & & & & & & \\
\hline $\mathrm{SBP}(\mathrm{mmHg})$ & $135 \cdot 8$ & $16 \cdot 7$ & $136 \cdot 3$ & $17 \cdot 2$ & $133 \cdot 1$ & 13.5 & 0.032 \\
\hline $\mathrm{DBP}(\mathrm{mmHg})$ & 79.0 & 11.2 & 78.5 & 11.3 & $82 \cdot 0$ & 10.5 & 0.001 \\
\hline MAP $(\mathrm{mmHg})$ & 98.0 & $11 \cdot 2$ & $97 \cdot 8$ & 11.4 & $99 \cdot 2$ & $10 \cdot 0$ & 0.227 \\
\hline \multicolumn{8}{|l|}{ Laboratory and nutrition data } \\
\hline Albumin $(g / l)$ & $35 \cdot 4$ & $4 \cdot 6$ & $35 \cdot 0$ & 4.5 & $37 \cdot 1$ & 4.6 & $<0.001$ \\
\hline $\mathrm{Hb}(\mathrm{g} / \mathrm{l})$ & $102 \cdot 8$ & $15 \cdot 6$ & $101 \cdot 7$ & $15 \cdot 6$ & $107 \cdot 9$ & $14 \cdot 9$ & $<0.001$ \\
\hline hs-CRP (mg/l) & & & & & & & \\
\hline Median & & & & & & & 0.001 \\
\hline Upper and lower quartiles & & & & & & & \\
\hline Urea $\mathrm{N}(\mathrm{mmol} / \mathrm{l})$ & 22.5 & $6 \cdot 1$ & $22 \cdot 3$ & $6 \cdot 0$ & 23.6 & $6 \cdot 8$ & 0.015 \\
\hline Serum creatinine $(\mu \mathrm{mol} / \mathrm{l})$ & 689.2 & 233.6 & $678 \cdot 2$ & $232 \cdot 6$ & $742 \cdot 2$ & $232 \cdot 2$ & 0.002 \\
\hline Serum Ca (mmol/l) & $2 \cdot 2$ & 0.2 & $2 \cdot 2$ & 0.2 & $2 \cdot 2$ & 0.2 & 0.535 \\
\hline Serum $\mathrm{P}(\mathrm{mmol} / \mathrm{l})$ & 1.6 & 0.4 & 1.6 & 0.4 & 1.6 & 0.4 & 0.908 \\
\hline Serum K $(\mathrm{mmol} / \mathrm{l})$ & 4.4 & 0.6 & 4.4 & 0.6 & 4.5 & 0.6 & 0.057 \\
\hline Serum $\mathrm{Na}(\mathrm{mmol} / \mathrm{l})$ & 139.2 & 3.0 & 139.0 & 3.1 & 139.8 & $2 \cdot 3$ & 0.007 \\
\hline HDL-cholesterol (mmol/l) & $1 \cdot 1$ & 0.3 & $1 \cdot 1$ & 0.3 & $1 \cdot 2$ & 0.3 & 0.240 \\
\hline LDL-cholesterol (mmol/l) & $2 \cdot 6$ & 0.8 & $2 \cdot 6$ & 0.8 & $2 \cdot 7$ & 0.7 & 0.261 \\
\hline Total cholesterol $(\mathrm{mmol} / \mathrm{l})$ & 4.9 & 1.1 & 4.9 & 1.2 & $5 \cdot 0$ & 1.0 & 0.255 \\
\hline TAG (mmol/l) & & & & & & & \\
\hline Median & & & & & & & 0.459 \\
\hline Upper and lower quartiles & & & & & & & \\
\hline iPTH (pg/ml) & & & & & & & \\
\hline Median & & & & & & & 0.441 \\
\hline Upper and lower quartiles & & & & & & & \\
\hline Total CCr (litres/week per $1.73 \mathrm{~m}^{2}$ ) & $72 \cdot 8$ & $27 \cdot 9$ & $72 \cdot 9$ & 28.5 & $72 \cdot 4$ & $25 \cdot 2$ & 0.831 \\
\hline Total Kt/N & 1.9 & 0.5 & 1.9 & 0.5 & $2 \cdot 0$ & 0.6 & 0.014 \\
\hline $\operatorname{RRF}(\mathrm{ml} / \mathrm{min})$ & & & & & & & \\
\hline Median & & & & & & & 0.139 \\
\hline Upper and lower quartiles & & & & & & & \\
\hline Total energy intake $(\mathrm{kJ} / \mathrm{d})$ & $6949 \cdot 2$ & 1398.7 & 6233.3 & 1564.8 & $7040 \cdot 4$ & $1228 \cdot 8$ & $<0.001$ \\
\hline Total protein intake $(\mathrm{g} / \mathrm{d})$ & $52 \cdot 1$ & 13.9 & $51 \cdot 6$ & $14 \cdot 2$ & $54 \cdot 4$ & $12 \cdot 5$ & 0.028 \\
\hline Total carbohydrate intake $(\mathrm{g} / \mathrm{d})$ & $186 \cdot 7$ & $52 \cdot 4$ & $184 \cdot 2$ & $54 \cdot 1$ & $196 \cdot 8$ & $44 \cdot 2$ & 0.009 \\
\hline Total fat intake (g/d) & $54 \cdot 0$ & 14.5 & $53 \cdot 0$ & 14.7 & $58 \cdot 2$ & 13.7 & $<0.001$ \\
\hline Total fibre intake $(\mathrm{g} / \mathrm{d})$ & $8 \cdot 2$ & $3 \cdot 4$ & 8.0 & 3.3 & $9 \cdot 1$ & $3 \cdot 3$ & $<0.001$ \\
\hline $\mathrm{nDPI}(\mathrm{g} / \mathrm{kg}$ per $\mathrm{d})$ & 0.85 & 0.2 & 0.82 & 0.2 & 0.93 & 0.2 & $<0.001$ \\
\hline $\mathrm{nDEl}(\mathrm{kJ} / \mathrm{kg}$ per $\mathrm{d})$ & $119 \cdot 2$ & $22 \cdot 6$ & $117 \cdot 6$ & 23.8 & $125 \cdot 9$ & $19 \cdot 2$ & $<0.001$ \\
\hline
\end{tabular}

DM, diabetes mellitus; SBP, systolic blood pressure; DBP, diastolic blood pressure; MAP, mean arterial pressure; hs-CRP, high-sensitive C-reactive protein; iPTH, intact parathyroid hormone; Total CCr, total creatinine clearance; Total Kt/V, total urea clearance; RRF, residual renal function; nDPI, normalised protein intake; nDEI, normalised energy intake.

measurements. And we chose the 3-year period of observation here to calculate the total time-averaged values. All statistical tests were two tailed, and the significance level was set at $P<0.05$.

\section{Results}

\section{Subject demographics and follow-up}

We followed 881 incident PD patients ( 434 men and 447 women), mean age of 57.7 (SD 14.8) years for 45.0 (interquartile range $21 \cdot 5,74 \cdot 0)$ months; $42 \cdot 1 \%$ had DM, and CVD was present in $42.6 \%$ (Table 1 ).

At the end of the study, 151 patients were still being maintained on PD, 434 had died, 164 were transferred to HD, 114 had undergone renal transplantation and eighteen were lost to follow-up (Fig. 1). A total of 178 (41.0 \%) of 434 of all deaths were due to cardiovascular causes, and 107 (24.7\%) of 434 were due to infection (Table 3). According to the time-averaged fibre intake, patients in the lower tertile had a much shorter followup time and higher mortality rate $(P<0.001)$. Along with an increased intake of fibre, patients were less prone to be 


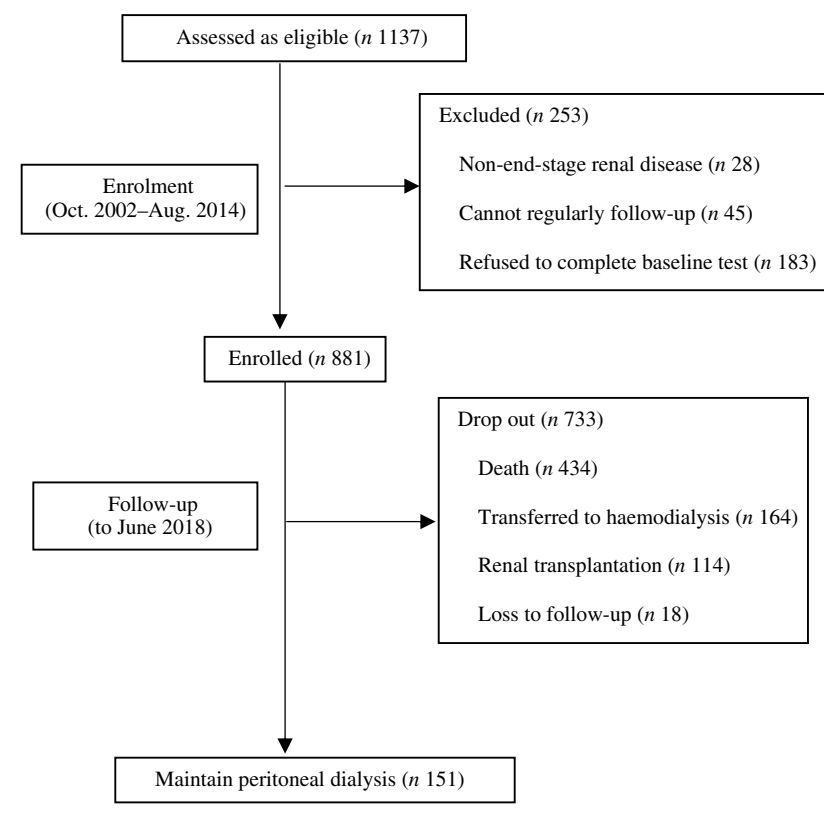

Fig. 1. Flow chart of the study.

transferred to HD due to socio-economic causes and more likely to receive the renal transplantation. The main characteristics of ceased and alive patients are presented in Table 2.

\section{Dietary fibre intake and clinical characteristics at baseline}

The baseline fibre intake was 8.2 (sD 3.4) g/d in our cohort. The baseline characteristics of the study population tertiled by baseline fibre intake are given in Table 1. Significant differences were found between groups with regard to age; sex; BMI; the prevalence of CVD; diastolic blood pressure (DBP) and mean arterial pressure; Alb; Hb; hs-CRP; urea N; K; Na; total cholesterol; TAG; iPTH; RRF; total creatinine clearance; dietary nutrients including energy, protein, carbohydrate, fat, fibre, normalised daily protein and energy intake levels $(P<0.001,0.01$ or 0.05$)$. Patients in the middle or/and high tertile were more likely to be male, younger, have higher BMI and low prevalence of CVD than those in the low tertile. The levels of DBP, mean arterial pressure, $\mathrm{Alb}, \mathrm{Hb}, \mathrm{K}, \mathrm{Na}$, iPTH and all dietary nutrients in the middle or/ and high tertile were also significantly higher than those in the low tertile. In addition, patients in the middle or/and high tertile had better RRF and serum lipid spectrum and lower inflammation level.

\section{Dietary fibre intake and the change in trend of blood pressure and biochemistry data during follow-up}

The change in trend of blood pressure and all laboratory measurements was explored according to the tertile of the timeaveraged dietary fibre intake adjusted for age, sex and BMI. The trends of DBP, serum Alb, $\mathrm{Hb}$ and hs-CRP were significantly different between tertiles $(P<0 \cdot 001)$. The high or/and middle fibre intake group had increased DBP values compared with low-fibre intake group during the first 24-month or increased $\mathrm{Hb}$ values during the first 12-month observation period $(P<0.05)$ (Fig. 2(b) and (c)). The serum Alb values increased with increased intake of fibre at each time point during the whole period $(P<0.05)$ (Fig. 2(d)). Besides, hs-CRP values in low-fibre intake group were significantly higher than those in the highfibre intake group during the whole period and also were higher than those in the middle group after 6 months $(P<0.05)$ (Fig. 2(f)).

No differences were observed between groups with regard to the change in trend of systolic blood pressure, RRF, TAG and cholesterol between groups (Fig. 2(a), (e), (g) and (h)). In addition, other laboratory measurements such as serum $\mathrm{Ca}, \mathrm{P}, \mathrm{K}, \mathrm{Na}$ and iPTH did not show significant differences during the follow-up between groups (data not shown).

\section{Predictive value of fibre intake for all-cause and CVD mortality}

The relationship between baseline or time-averaged fibre intake and outcomes was analysed. The baseline and time-averaged fibre intake were significantly associated with lower all-cause mortality in the unadjusted analysis (HR 0.82 (95\% CI 0.78 , 0.85), $P<0.001$ and HR 0.87 (95\% CI 0.84, 0.90), $P<0.001$ ). By multivariate Cox regression analysis, with an increase of 1 $\mathrm{g} / \mathrm{d}$ in baseline and time-averaged dietary fibre intake, the allcause mortality showed a trend to decrease by $4 \%(P=0.053)$ and $6 \%(P=0.065)$, respectively, not achieving significant differences after multivariate adjustment. For subgroups analyses, an increase of $1 \mathrm{~g} / \mathrm{d}$ in time-averaged fibre intake was significantly associated with a $13 \%$ decrease in all-cause mortality (HR 0.87 (95 \% CI 0.76, 0.98), $P=0.022$ ) among non-diabetic patients (Table 4).

When our subjects were categorised by baseline or timeaveraged dietary fibre, we did not find that high-tertile group showing any protective effects on the all-cause mortality compared with the low-tertile group. A trend of benefits for high tertile of fibre intake in all-cause mortality were found among patients without CVD, but not achieving significant differences.

As for CVD mortality, only the baseline fibre intake showed a protective effect (HR 0.94 (95\% CI 0.89, 0.99), $P=0.019$ ) in the unadjusted analysis. Based on multivariate Cox regression analysis, no obvious association was found between dietary fibre intake as continuous or categorical variables and CVD mortality, either in the whole cohort or in other subgroups (Table 5).

\section{Discussion}

In this long-term prospective cohort study, participants with higher fibre intake were more likely to be younger and male. They tended to have better nutritional status, higher blood pressure and lower inflammatory status at baseline and afterward. Both baseline and time-averaged fibre intake showed protective effects against all-cause mortality by univariate analysis. Despite that this benefit was weakened after multivariate adjustment for the whole cohort, an independent association between fibre intake and all-cause mortality was still found among nondiabetic patients on PD.

An inverse association between dietary fibre intake and allcause mortality is considered plausible. Some clinical clues should be recognised from the present study. Patients with 
(a)

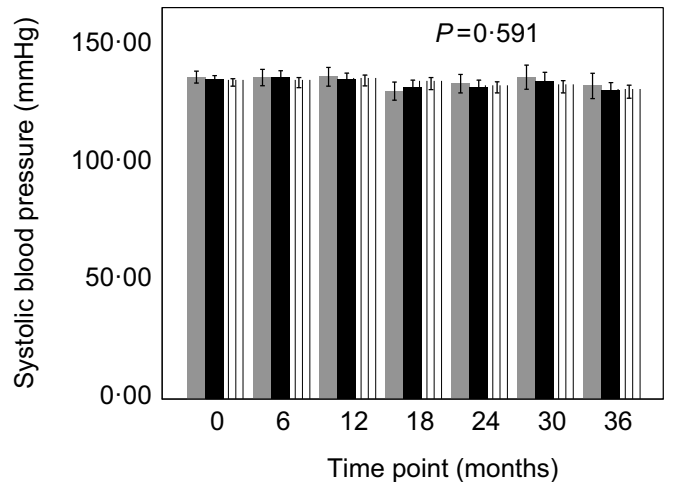

(c)

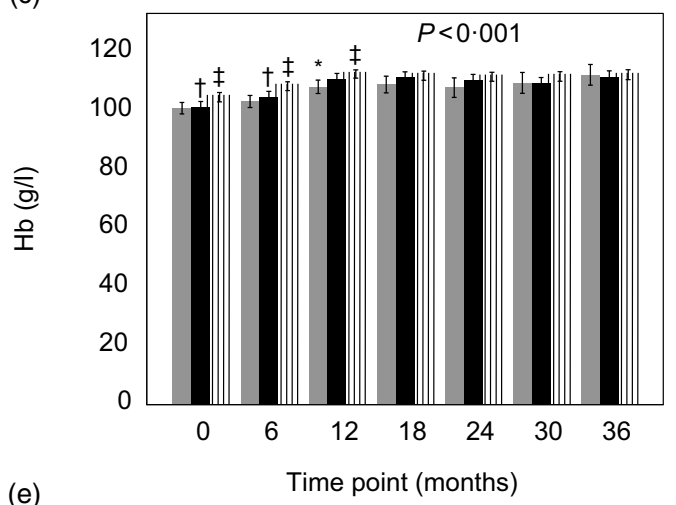

(e)

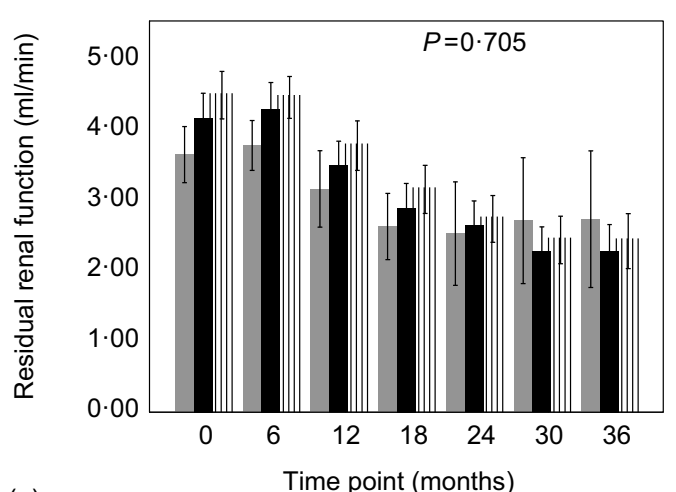

(g)

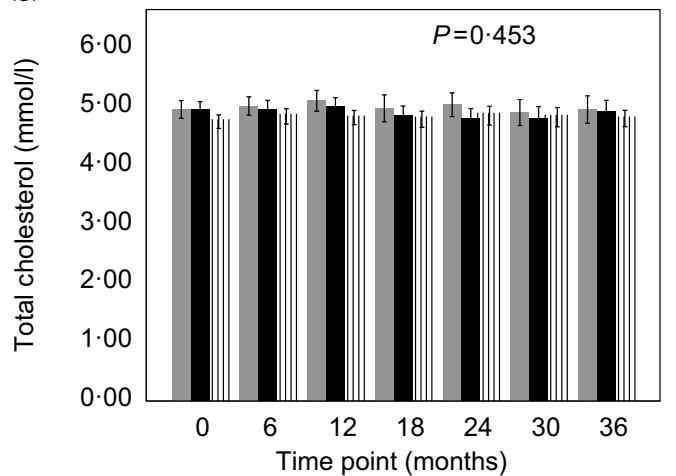

(b)

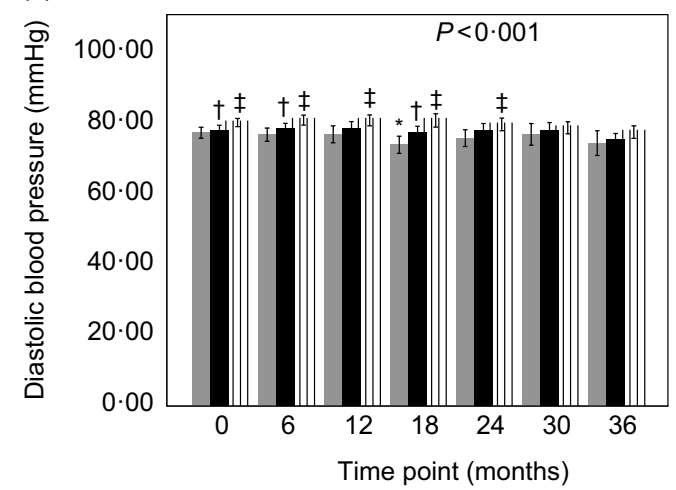

(d)
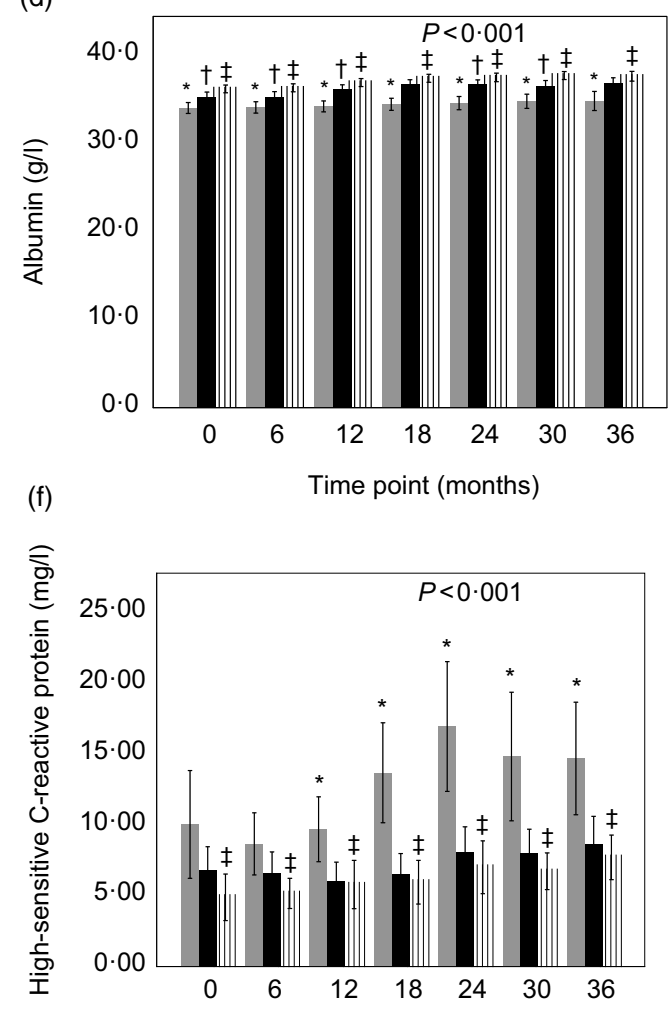

(h)



Fig. 2. Blood pressure, renal function and laboratory data of peritoneal dialysis patients with different tertiles of time-averaged fibre intake (๓, low tertile; $\mathbf{m}$, middle tertile; IIII, high tertile) observed for a period of 36 months. Values are means, and lower and upper bar boundaries are $95 \%$ confidence intervals. $P$ value comparing the three groups over time was obtained from the linear mixed model with bootstrap covariance accounting for correlated measures within a subject adjusted for baseline characteristics, age, sex and BMI. * $P<0.05$ low-tertile group compared with the middle-tertile group at the same time point. $\dagger P<0.05$ middle-tertile group compared with the high-tertile group at the same time point. $\ddagger P<0.05$ high-tertile group compared with the low-tertile group at the same time point. 
Table 3. Outcomes among peritoneal dialysis (PD) patients ( $n$ 881)

(Medians and interquartile ranges; number of events and event rate/100 person-years)

\begin{tabular}{|c|c|c|c|c|c|c|c|c|c|}
\hline \multirow[b]{3}{*}{ Outcomes } & \multirow{2}{*}{\multicolumn{2}{|c|}{ Total }} & \multicolumn{6}{|c|}{ Tertile of time-averaged fibre intake } & \multirow[b]{3}{*}{$P$} \\
\hline & & & \multicolumn{2}{|c|}{$\begin{array}{c}\text { Low } \\
(<6.2 \mathrm{~g} / \mathrm{d})\end{array}$} & \multicolumn{2}{|c|}{$\begin{array}{c}\text { Middle } \\
(6.2-8.3 \mathrm{~g} / \mathrm{d})\end{array}$} & \multicolumn{2}{|c|}{$\begin{array}{c}\text { High } \\
(>8.3 \mathrm{~g} / \mathrm{d}) \\
\end{array}$} & \\
\hline & $\begin{array}{l}\text { No. of } \\
\text { events }\end{array}$ & $\begin{array}{l}\text { Event rate/100 } \\
\text { person-years }\end{array}$ & $\begin{array}{l}\text { No. of } \\
\text { events }\end{array}$ & $\begin{array}{c}\text { Event rate/100 } \\
\text { person-years }\end{array}$ & $\begin{array}{l}\text { No. of } \\
\text { events }\end{array}$ & $\begin{array}{l}\text { Event rate/100 } \\
\text { person-years }\end{array}$ & $\begin{array}{l}\text { No. of } \\
\text { events }\end{array}$ & $\begin{array}{c}\text { Event rate/100 } \\
\text { person-years }\end{array}$ & \\
\hline \multicolumn{10}{|l|}{ Follow-up (months) } \\
\hline Median & \multirow{2}{*}{\multicolumn{2}{|c|}{$\begin{array}{c}45 \cdot 0 \\
21 \cdot 5,74 \cdot 0\end{array}$}} & \multirow{2}{*}{\multicolumn{2}{|c|}{$\begin{array}{c}33 \cdot 0^{\star} \\
17 \cdot 0,62 \cdot 0\end{array}$}} & \multirow{2}{*}{\multicolumn{2}{|c|}{$\begin{array}{c}47 \\
22 \cdot 0,75 \cdot 0\end{array}$}} & \multirow{2}{*}{\multicolumn{2}{|c|}{$\begin{array}{c}54 \cdot 0 \ddagger \\
25 \cdot 8,81 \cdot 0\end{array}$}} & $<0.001$ \\
\hline Interquartile range & & & & & & & & & \\
\hline Death & 434 & 11.52 & $186^{*}$ & $18 \cdot 08$ & $148 \dagger$ & 11.00 & $100 \ddagger$ & $7 \cdot 17$ & $<0.001$ \\
\hline Cardiovascular events§ & 178 & 4.72 & 68 & $6 \cdot 61$ & 67 & 4.98 & 43 & 3.08 & $0 \cdot 138$ \\
\hline Infection & 107 & 2.84 & 41 & 3.99 & 37 & $2 \cdot 75$ & 29 & $2 \cdot 08$ & $0 \cdot 279$ \\
\hline Transfer to haemodialysis & 164 & 4.35 & 45 & 4.38 & 53 & 3.94 & 66 & 4.73 & 0.554 \\
\hline PD-related infection & 95 & $2 \cdot 52$ & 23 & $2 \cdot 24$ & 34 & 2.53 & 38 & $2 \cdot 73$ & 0.378 \\
\hline Renal transplantation & 114 & 3.03 & 25 & 2.43 & $35 \dagger$ & $2 \cdot 60$ & $54 \ddagger$ & 3.87 & 0.031 \\
\hline
\end{tabular}

* $P<0.05$ low-tertile group compared with middle-tertile group.

$\dagger P<0.05$ middle-tertile group compared with high-tertile group.

$\ddagger P<0.05$ high-tertile group compared with low-tertile group.

$\S$ Cardiovascular events include cardiovascular events, cerebrovascular events and sudden death

higher fibre intake were likely to eat more food enriched with all dietary nutrients and thus maintain high values of serum Alb and $\mathrm{Hb}$ during the study period. Since serum Alb is significantly predictive of clinical outcomes ${ }^{(23)}$, an increase of approximate $2 \mathrm{~g} / \mathrm{l}$ in serum Alb in the high tertile might have substantial contribution to the benefits of dietary fibre intake on mortality, as shown in our data. In addition, serum CRP levels decreased along with the increased dietary fibre across the tertiles during the whole period. Similarly, the NHANES III study reported that the odds of elevated serum CRP levels decreased by $38 \%$ in subjects with CKD for each $10-\mathrm{g} / \mathrm{d}$ increase in total fibre intake ${ }^{(11)}$. The ULSAM cohort study also showed that the odds of having CRP $>3 \mathrm{mg} / \mathrm{l}$ were lower in the quartiles that consumed more fibre ${ }^{(10)}$. The causes underlying the relationship between dietary fibre and inflammation have been explored recently. A high-fibre diet could alter gut bacterial metabolism and decrease the generation and absorption of some toxins that trigger systemic inflammation $^{(24)}$. A high-fibre diet also has been proposed to be associated with a lower glycaemic load of rapidly digestible and absorbable dietary carbohydrates ${ }^{(25,26)}$ and higher plasma levels of the antiinflammatory protein adiponectin ${ }^{(27)}$. In addition, fibres may provide a number of health benefits on the kidney by selectively stimulating favourable growth or activity of a limited number of indigenous bacteria to alter the composition of the intestinal flora and promote toxin discharge, improving the lipid metabolism ${ }^{(28)}$. Since dietary fibre could exert multifactorial effects for CKD and PD patients, we should draw more attention to observe the potential benefits of increasing fibre intake via dietary or supplements in this population.

Previous studies in the general population suggested that dietary fibre is predictive of lower blood pressure ${ }^{(29)}$, which might be due to the improved postprandial glucose excursions, insulin resistance ${ }^{(30)}$ and endothelial dysfunction ${ }^{(31)}$. Our data indicated a positive association of fibre intake and DBP at baseline and during follow-up. This paradoxical phenomenon might be explained as follows. Our subjects in the high-tertile fibre group had a significantly higher nutrient intake. We cannot exclude the possibility that other nutrients, such as Na, phosphate and water, exerted harmful effects on blood pressure ${ }^{(32,33)}$, offsetting the benefits of dietary fibre. Besides, dietary fibre has many types, such as chitosan, mannans and pectins, which may exert different effects on the blood pressure ${ }^{(34,35)}$, and an overall analysis of the total dietary fibre intake cannot determine the effects in detail.

In the present study, the amounts of time-averaged dietary fibre intake were markedly low, that is, 4.6, 6.8 and $10 \cdot 1 \mathrm{~g}$ in the three tertiles, respectively, which are relatively lower than $10 \cdot 2-12 \cdot 4 \mathrm{~g} / \mathrm{d}$ of fibre in HD patients ${ }^{(22,36)}$, and $19 \cdot 8 \mathrm{~g} / \mathrm{d}$ of fibre in non-dialysed patients with $\mathrm{CKD}^{(37)}$. All the above-mentioned fibre intake levels among CKD patients are lower than the target set for healthy individuals ${ }^{(38-40)}$. Indeed, patients in the late stage of CKD, with the loss of renal function, were requested to decrease the intake of dietary $\mathrm{K}$, phosphate and fluid. Their dietary pattern may lead to a simultaneous reduction in dietary fibre due to the restriction on vegetables, fruit and nuts. Unfortunately, current guidelines provide inconsistent references for dietary fibre intake for CKD patients ${ }^{(41)}$. The Kidney Disease: Improving Global Outcomes (KDIGO) Clinical Practice Guidelines, European Society for Clinical Nutrition and Metabolism (ESPEN) guidelines and Kidney Health Australia - Caring for Australasians with Renal Impairment (KHA-CARI) guidelines emphasise the inclusion of fibre-rich foods in the diet or a balanced diet rich in fruit and vegetables but do not recommend an exact amount for fibre ${ }^{(14-16)}$. The Kidney Disease Outcomes Quality Initiative (KDOQI) Guidelines on dyslipidaemia recommend $20-30 \mathrm{~g} / \mathrm{d}$ of fibre ${ }^{(42)}$. In view of limited data in the field, it is necessary to call for robust evidence regarding the appropriate quantity of dietary fibre in non-dialysed and dialysed CKD populations.

The present study had several strengths. To the best of our knowledge, it is the first study to explore whether the baseline and time-averaged fibre intake can predict all-cause mortality 
Table 4. Prognostic value of dietary fibre for all-cause mortality (Hazard ratios (HR) and $95 \%$ confidence intervals)

\begin{tabular}{|c|c|c|c|c|c|c|c|c|c|c|c|c|c|c|c|c|}
\hline \multirow{2}{*}{ Fibre intake } & & \multicolumn{3}{|c|}{ Total $(n 881)$} & \multicolumn{3}{|c|}{$\mathrm{DM}(n 371)$} & \multicolumn{3}{|c|}{ Non-DM $(n 510)$} & \multicolumn{3}{|c|}{ CVD (n 375) } & \multicolumn{3}{|c|}{ Non-CVD ( $n$ 509) } \\
\hline & & $\mathrm{HR}$ & $95 \% \mathrm{Cl}$ & $P$ & $\mathrm{HR}$ & $95 \% \mathrm{Cl}$ & $P$ & $\mathrm{HR}$ & $95 \% \mathrm{Cl}$ & $P$ & HR & $95 \% \mathrm{Cl}$ & $P$ & $\mathrm{HR}$ & $95 \% \mathrm{Cl}$ & $P$ \\
\hline \multicolumn{17}{|l|}{ Continuous variables } \\
\hline Baseline* $^{*}$ & & 0.96 & $0.91,1.00$ & 0.053 & 0.95 & $0.89,1.01$ & 0.114 & 0.97 & $0.91,1.03$ & 0.313 & 0.98 & $0.93,1.04$ & 0.569 & 0.96 & $0.89,1.03$ & 0.238 \\
\hline Time averaged $\dagger$ & & 0.94 & $0.87,1.00$ & 0.065 & 0.97 & $0.90,1.05$ & 0.505 & 0.87 & $0.76,0.98$ & 0.022 & 0.48 & $0.87,1.07$ & 0.479 & 0.96 & $0.86,1.06$ & 0.415 \\
\hline \multicolumn{17}{|l|}{ Categorical variables } \\
\hline Baseline* & Tertile 1 & & Reference & & & Reference & & & Reference & & & Reference & & & Reference & \\
\hline & Tertile 2 & 0.85 & $0.65,1 \cdot 10$ & 0.207 & 0.92 & $0.64,1.33$ & 0.653 & 0.86 & $0.58,1.27$ & 0.453 & 0.95 & $0.67,1.33$ & 0.749 & 0.77 & $0.50,1.17$ & 0.215 \\
\hline & Tertile 3 & 0.82 & $0.60,1 \cdot 14$ & 0.236 & 0.80 & $0.52,1.22$ & 0.299 & 0.86 & $0.53,1.41$ & 0.553 & $1 \cdot 12$ & $0.75,1.68$ & 0.588 & 0.61 & $0.36,1.04$ & 0.070 \\
\hline \multirow[t]{3}{*}{ Time averaged $\dagger$} & Tertile 1 & & Reference & & & Reference & & & Reference & & & Reference & & & Reference & \\
\hline & Tertile 2 & 0.75 & $0.56,1.02$ & 0.064 & 0.81 & $0.53,1.23$ & 0.324 & 0.71 & $0.44,1.14$ & 0.160 & 0.77 & $0.51,1.15$ & 0.201 & 0.85 & $0.53,1.37$ & 0.504 \\
\hline & Tertile 3 & 0.71 & $0.48,1.05$ & 0.084 & 0.91 & $0.53,1.55$ & 0.722 & 0.58 & $0.31,1.08$ & 0.085 & 1.04 & $0.60,1.78$ & 0.902 & 0.66 & $0.35,1.23$ & 0.189 \\
\hline
\end{tabular}

DM, diabetes mellitus; MAP, mean arterial pressure; hs-CRP, high-sensitive C-reactive protein; iPTH, intact parathyroid hormone; RRF, residual renal function.

${ }^{*}$ Adjusted for age, sex, BMI, MAP, Hb, albumin, hs-CRP, TAG, iPTH, RRF, total protein intake, total energy intake.

† Adjusted for age, sex, time-averaged BMI, time-averaged MAP, time-averaged $\mathrm{Hb}$, time-averaged albumin, time-averaged hs-CRP, time-averaged TAG, time-averaged iPTH, time-averaged RRF, time-averaged total protein intake, time-

averaged total energy intake.

Table 5. Prognostic value of dietary fibre for CVD mortality

(Hazard ratios (HR) and $95 \%$ confidence intervals)

\begin{tabular}{|c|c|c|c|c|c|c|c|c|c|c|c|c|c|c|c|c|}
\hline \multirow[b]{2}{*}{ Fibre intake } & & \multicolumn{3}{|c|}{ Total $(n 881)$} & \multicolumn{3}{|c|}{$\mathrm{DM}(n 371)$} & \multicolumn{3}{|c|}{ Non-DM ( $n$ 510) } & \multicolumn{3}{|c|}{ CVD ( $n$ 375) } & \multicolumn{3}{|c|}{ Non-CVD ( $n$ 509) } \\
\hline & & $\mathrm{HR}$ & $95 \% \mathrm{Cl}$ & $P$ & $\mathrm{HR}$ & $95 \% \mathrm{Cl}$ & $P$ & HR & $95 \% \mathrm{Cl}$ & $P$ & $\mathrm{HR}$ & $95 \% \mathrm{Cl}$ & $P$ & $\mathrm{HR}$ & $95 \% \mathrm{Cl}$ & $P$ \\
\hline \multicolumn{17}{|l|}{ Continuous variables } \\
\hline Baseline $^{*}$ & & 0.95 & $0.88,1.02$ & 0.166 & 0.94 & $0.85,1.05$ & 0.288 & 0.96 & $0.85,1.07$ & 0.441 & 0.99 & $0.90,1.08$ & 0.820 & 0.94 & $0.79,1.12$ & 0.511 \\
\hline Time averaged $\dagger$ & & 0.97 & $0.87,1.08$ & 0.590 & 1.01 & $0.90,1.13$ & 0.868 & 0.85 & $0.68,1.05$ & 0.126 & 0.94 & $0.79,1.12$ & 0.497 & 1.05 & $0.94,1.18$ & 0.362 \\
\hline \multicolumn{17}{|l|}{ Categorical variables } \\
\hline Baseline* & Tertile 1 & & Reference & & & Reference & & & Reference & & & Reference & & & Reference & \\
\hline & Tertile 2 & 0.99 & $0.65,1.51$ & 0.956 & 1.26 & $0.71,2.23$ & 0.432 & 0.86 & $0.44,1.68$ & 0.663 & 1.07 & $0.64,1.80$ & 0.794 & 0.94 & $0.39,2.25$ & 0.891 \\
\hline & Tertile 3 & 0.83 & $0.51,1.37$ & 0.474 & 0.84 & $0.45,1.57$ & 0.584 & 0.74 & $0.31,1.77$ & 0.497 & $1 \cdot 12$ & $0.64,1.97$ & 0.688 & 0.40 & $0.11,1.41$ & 0.154 \\
\hline \multirow[t]{3}{*}{ Time averaged $\dagger$} & Tertile 1 & & Reference & & & Reference & & & Reference & & & Reference & & & Reference & \\
\hline & Tertile 2 & 0.73 & $0.46,1.17$ & 0.193 & 0.53 & $0.27,1.04$ & 0.065 & 0.89 & $0.38,2.06$ & 0.785 & 0.76 & $0.41,1.41$ & 0.388 & 0.81 & $0.32,2.08$ & 0.662 \\
\hline & Tertile 3 & 0.68 & $0.37,1.26$ & 0.217 & 0.87 & $0.38,2.00$ & 0.740 & 0.50 & $0.16,1.54$ & 0.229 & 0.93 & $0.42,2.05$ & 0.849 & 0.66 & $0.21,2.12$ & 0.487 \\
\hline
\end{tabular}

DM, diabetes mellitus; MAP, mean arterial pressure; hs-CRP, high-sensitive C-reactive protein; iPTH, intact parathyroid hormone; RRF, residual renal function

* Adjusted for age, sex, BMI, MAP, Hb, albumin, hs-CRP, TAG, iPTH, RRF, total protein intake, total energy intake.

† Adjusted for age, sex, time-averaged BMI, time-averaged MAP, time-averaged $\mathrm{Hb}$, time-averaged albumin, time-averaged hs-CRP, time-averaged TAG, time-averaged iPTH, time-averaged RRF, time-averaged total protein intake, timeaveraged total energy intake. 
in patients on PD. We collected repeat measurements for dietary nutrients rather than a single-point assessment. The patients were thoroughly examined using time-averaged parameters for their dietary record, biochemical and nutrition data, giving us a unique chance to test our hypothesis. Oral nutrition supplements were also taken into account when evaluating the dietary nutrients. Furthermore, the study was performed in a large PD cohort with a relatively long follow-up and sufficient endpoints. We adjusted for total protein and energy intake when analysing the correlation between fibre and outcomes to minimise the confounding influences of these nutrients.

In spite of those strengths, the results of the present study should be interpreted in caution. First, an observational design cannot establish the cause-effect relationship between dietary intake and outcomes such as survival, serum Alb and hs-CRP. We cannot exclude that the inverse association of dietary fibre intake and all-cause and CVD mortality is confounded by physical activity or other healthy habits. More biological properties of dietary fibre, including its effect on insulin sensitivity, oxidative stress, inflammation and protein-bound uremic toxins, need to be investigated to explain the observed phenomenon in the dialysis population ${ }^{(43-45)}$. Moreover, dietary fibre was not distinguished by soluble and insoluble fibre. It was not determined whether different sources and types of fibre exert varied effects on clinical outcome. Last, the present study was conducted in a single centre, which limits the generalisability of our data.

Our study revealed that higher dietary fibre intake appeared to have a protective effect on all-cause mortality in non-diabetic PD patients, which suggests that PD patients should be encouraged to consume a fibre-rich diet. Following the observation that PD patients with higher fibre intake concomitantly have better nutrition status and lower inflammation, more potential mechanisms for the benefits of dietary fibre in traditional and non-traditional risk factors need to be explored. Interventional studies are warranted to determine the efficacy and feasibility of fibre intake by dietary or oral supplements in PD patients. Targets for dietary fibre intake should also be determined for patients with CKD.

\section{Acknowledgements}

The authors express their appreciation to the patients and staff of the Peritoneal Dialysis Center of Peking University First Hospital, for their continuing contribution to the present study. This work is supported in part by Capital Characteristic Clinic Research Grant from Beijing Science \& Technology Committee (Z111107058811110), New Century Excellent Talents from Education Department of China, Clinic Research Award from ISN GO R\&P Committee.

This research received no specific grant from any funding agency, commercial or not-for-profit sectors.

The contributions of the authors are as follows. Research idea and study design: J. D.; data acquisition: Z. L., Y. C., H. L.; statistical analysis: X. X., Z. L.; manuscript drafting or revision: X. X., J. D.; supervision or mentorship: J. D. Each author accepts accountability for the overall work by ensuring that questions pertaining to the accuracy or integrity of any portion of the work are appropriately investigated and resolved. J. D. takes responsibility that the present study has been reported honestly, accurately and transparently; that no important aspects of the study have been omitted and that any discrepancies from the study as planned have been explained. All authors read and approved the final manuscript.

There were no conflicts of interest.

\section{References}

1. Bradbury KE, Appleby PN \& Key TJ (2014) Fruit, vegetable, and fiber intake in relation to cancer risk: findings from the European Prospective Investigation into Cancer and Nutrition (EPIC). Am J Clin Nutr 100, Suppl. 1, 394s-398s.

2. Streppel MT, Arends LR, Veer PVT, et al. (2005) Dietary fiber and blood pressure: a meta-analysis of randomized placebocontrolled trials. Arch Intern Med 165, 150-156.

3. Aune D, Chan DS, Greenwood DC, et al. (2012) Dietary fiber and breast cancer risk: a systematic review and meta-analysis of prospective studies. Ann Oncol 23, 1394.

4. Threapleton DE, Greenwood DC, Evans CEL, et al. (2013) Dietary fibre intake and risk of cardiovascular disease: systematic review and meta-analysis. BMJ 347, f6879.

5. Mirmiran P, Yuzbashian E, Asghari G, et al. (2018) Dietary fibre intake in relation to the risk of incident chronic kidney disease. BrJ Nutr 119, 479-485.

6. Cho SS, Qi L, Fahey GCJr, et al. (2013) Consumption of cereal fiber, mixtures of whole grains and bran, and whole grains and risk reduction in type 2 diabetes, obesity, and cardiovascular disease. Am J Clin Nutr 98, 594-619.

7. Tanaka S, Yoshimura Y, Kamada C, et al. (2013) Intakes of dietary fiber, vegetables, and fruits and incidence of cardiovascular disease in Japanese patients with type 2 diabetes. Diabetes Care 36, 3916-3922.

8. Kim Y \& Je Y (2016) Dietary fibre intake and mortality from cardiovascular disease and all cancers: a meta-analysis of prospective cohort studies. Arch Cardiovasc Dis 109, 39-54.

9. Yao B, Fang H, Xu W, et al. (2014) Dietary fiber intake and risk of type 2 diabetes: a dose-response analysis of prospective studies. Eur J Epidemiol 29, 79-88.

10. Xu H, Huang X, Riserus U, et al. (2014) Dietary fiber, kidney function, inflammation, and mortality risk. Clin J Am SOC Nephrol 9, 2104-2110.

11. Krishnamurthy VM, Wei G, Baird BC, et al. (2012) High dietary fiber intake is associated with decreased inflammation and allcause mortality in patients with chronic kidney disease. Kidney Int 81, 300-306.

12. Sutton D, Ovington S \& Engel B (2014) A multi-centre, randomised trial to assess whether increased dietary fibre intake (using a fibre supplement or high-fibre foods) produces healthy bowel performance and reduces laxative requirement in free living patients on peritoneal dialysis. J Ren Care $\mathbf{4 0}$, $157-163$.

13. Xie LM, Ge YY, Huang X, et al. (2015) Effects of fermentable dietary fiber supplementation on oxidative and inflammatory status in hemodialysis patients. Int J Clin Exp Med 8, 1363-1369.

14. Toigo G, Aparicio M, Attman PO, et al. (2000) Expert Working Group report on nutrition in adult patients with renal insufficiency (part 1 of 2). Clin Nutr 19, 197-207.

15. Johnson DW, Atai E, Chan M, et al. (2013) KHA-CARI guideline: early chronic kidney disease: detection, prevention and management. Nephrology (Carlton) 18, 340-350.

16. Wheeler D \& Kasiske B (2013) KDIGO clinical practice guideline for the evaluation and management of chronic kidney disease foreword. Kidney Int Suppl 3, 2. 
17. Kelly JT, Palmer SC, Wai SN, et al. (2017) Healthy dietary patterns and risk of mortality and ESRD in CKD: a meta-analysis of cohort studies. Clin J Am Soc Nephrol 12, 272-279.

18. Smith SC Jr, Jackson R, Pearson TA, et al. (2004) Principles for national and regional guidelines on cardiovascular disease prevention: a scientific statement from the World Heart and Stroke Forum. Circulation 109, 3112-3121.

19. Dong J, Li Y, Xu Y, et al. (2011) Daily protein intake and survival in patients on peritoneal dialysis. Nephrol Dial Transplant 26, 3715-3721.

20. Dong J, Li Y, Yang Z, et al. (2010) Low dietary sodium intake increases the death risk in peritoneal dialysis. Clin J Am Soc Nephrol 5, 240-247.

21. Fassett RG, Robertson IK, Geraghty DP, et al. (2007) Dietary intake of patients with chronic kidney disease entering the LORD trial: adjusting for underreporting. J Ren Nutr 17, 235-242.

22. Kalantar-Zadeh K, Kopple JD, Deepak S, et al. (2002) Food intake characteristics of hemodialysis patients as obtained by food frequency questionnaire. J Ren Nutr 12, 17-31.

23. Friedman AN \& Fadem SZ (2010) Reassessment of albumin as a nutritional marker in kidney disease. J Am Soc Nephrol 21, 223-230.

24. Evenepoel P, Meijers BK, Bammens BR, et al. (2009) Uremic toxins originating from colonic microbial metabolism. Kidney Int Suppl 76, S12-S19.

25. Liu S, Manson JE, Buring JE, et al. (2002) Relation between a diet with a high glycemic load and plasma concentrations of high-sensitivity C-reactive protein in middle-aged women. Am J Clin Nutr 75, 492-498.

26. Qi L, van Dam RM, Liu S, et al. (2006) Whole-grain, bran, and cereal fiber intakes and markers of systemic inflammation in diabetic women. Diabetes Care 29, 207-211.

27. Qi L, Rimm E, Liu S, et al. (2005) Dietary glycemic index, glycemic load, cereal fiber, and plasma adiponectin concentration in diabetic men. Diabetes Care 28, 1022-1028.

28. Slavin J (2013) Fiber and prebiotics: mechanisms and health benefits. Nutrients 5, 1417-1435.

29. Khan K, Jovanovski E, Ho HVT, et al. (2018) The effect of viscous soluble fiber on blood pressure: a systematic review and meta-analysis of randomized controlled trials. Nutr Metab Cardiovasc Dis 28, 3-13.

30. Wong JMW \& Jenkins DJA (2007) Carbohydrate digestibility and metabolic effects. J Nutr 137, 11 Suppl., 2539 S.
31. Montero D (2013) Hemodynamic actions of insulin: beyond the endothelium. Front Physiol 4, 389.

32. Sanghavi S \& Vassalotti JA (2013) Dietary sodium: a therapeutic target in the treatment of hypertension and CKD.J Ren Nutr $\mathbf{2 3}$, 223-227.

33. Chan Q, Stamler J, Griep LM, et al. (2016) An update on nutrients and blood pressure. J Atheroscler Thromb 23, 276-289.

34. Evans CE, Greenwood DC, Threapleton DE, et al. (2015) Effects of dietary fibre type on blood pressure: a systematic review and meta-analysis of randomized controlled trials of healthy individuals. J Hypertens 33, 897-911.

35. Wood RJ, Fernandez ML, Sharman MJ, et al. (2007) Effects of a carbohydrate-restricted diet with and without supplemental soluble fiber on plasma low-density lipoprotein cholesterol and other clinical markers of cardiovascular risk. Metab Clin Exp 56, 58-67.

36. Khoueiry G, Waked A, Goldman M, et al. (2011) Dietary intake in hemodialysis patients does not reflect a heart healthy diet. J Ren Nutr 21, 438-447.

37. Fassett RG, Robertson IK, Geraghty DP, et al. (2007) Dietary intake of patients with chronic kidney disease entering the LORD trial: adjusting for underreporting. J Ren Nutr 17, 235-242.

38. Government of Canada (2007) Canada's Food Guide. Ottawa: Health Canada. https://food-guide.canada.ca/en/ (accessed August 2019).

39. Food Standards Agency (2007) United Kingdom Eatwell Plate, Food Standards Agency. https://www.food.gov.uk/ (accessed August 2019).

40. USDA and U.S. Department of Health and Human Services (2010) Dietary Guidelines for Americans, 2010, 7th ed. Washington, DC: U.S. Government Printing Office.

41. Sabatino A, Regolisti G, Gandolfini I, et al. (2017) Diet and enteral nutrition in patients with chronic kidney disease not on dialysis: a review focusing on fat, fiber and protein intake. J Nephrol 30, 743-754.

42. KDOQI (2007) KDOQI clinical practice guidelines and clinical practice recommendations for diabetes and chronic kidney disease. Am J Kidney Dis 49, 2 Suppl. 2, S12-S154.

43. Lattimer JM \& Haub MD (2010) Effects of dietary fiber and its components on metabolic health. Nutrients 2, 1266-1289.

44. Anderson JW, Baird P, Davis RH Jr, et al. (2009) Health benefits of dietary fiber. Nutr Rev 67, 188-205.

45. Rossi M, Johnson DW, Xu H, et al. (2015) Dietary protein-fiber ratio associates with circulating levels of indoxyl sulfate and p-cresyl sulfate in chronic kidney disease patients. Nutr Metab Cardiovasc Dis 25, 860-865. 